\title{
A new characterization of the Sobolev space
}

\author{
by \\ Piotr HajŁasz (Warszawa)
}

\begin{abstract}
The purpose of this paper is to provide a new characterization of the Sobolev space $W^{1,1}\left(\mathbb{R}^{n}\right)$. We also show a new proof of the characterization of the Sobolev space $W^{1, p}\left(\mathbb{R}^{n}\right), 1 \leq p<\infty$, in terms of Poincaré inequalities.
\end{abstract}

The Sobolev space $W^{1, p}\left(\mathbb{R}^{n}\right), 1 \leq p<\infty$, consists of functions $u \in$ $L^{p}\left(\mathbb{R}^{n}\right)$ such that $|\nabla u| \in L^{p}\left(\mathbb{R}^{n}\right)$. It is a Banach space with respect to the norm

$$
\|u\|_{1, p}=\|u\|_{p}+\|\nabla u\|_{p}
$$

Let us point out that from the point of view of Banach spaces the structure of various Sobolev type spaces, with the particular emphasis on $W^{1,1}$, has been investigated by A. Pełczyński, M. Wojciechowski and others; see e.g. [4], [40]-[43] and references therein.

The purpose of this paper is to provide a new characterization of the Sobolev space $W^{1,1}\left(\mathbb{R}^{n}\right)$. Here, however, we emphasize future applications in geometric analysis and analysis on metric spaces rather than the theory of Banach spaces. Actually one of the reasons for finding new characterizations of the Sobolev space is the development of analysis on metric spaces; see e.g. [1], [2], [5], [6], [12], [13], [15]-[19], [21], [23], [24], [28]-[31], [38], [47]-[49], [51]. More references will be given later. In order to define a Sobolev type space on a metric-measure space we need a characterization of the space $W^{1, p}\left(\mathbb{R}^{n}\right)$ that does not involve derivatives. One such characterization is given in the following result.

TheOREM 1 ([19]). $u \in W^{1, p}\left(\mathbb{R}^{n}\right), 1<p<\infty$, if and only if $u \in L^{p}\left(\mathbb{R}^{n}\right)$ and there exists $0 \leq g \in L^{p}\left(\mathbb{R}^{n}\right)$ such that

$$
|u(x)-u(y)| \leq|x-y|(g(x)+g(y)) \quad \text { a.e. }
$$

2000 Mathematics Subject Classification: Primary 46E35.

This work was supported by the KBN grant 2 PO3A 02822 . The research was completed during the stay of the author in Department of Mathematics at University of Michigan. The author wishes to thank the University for the support and hospitality. 
Moreover

$$
\|\nabla u\|_{p} \approx \inf _{g}\|g\|_{p},
$$

where the infimum is taken over the class of functions $g$ satisfying (1).

Inequality (1) holds a.e. in the sense that there is a set $E \subset \mathbb{R}^{n}$ of measure zero such that (1) holds for all $x, y \in \mathbb{R}^{n} \backslash E$. Writing $A \approx B$ we mean that there is a constant $C \geq 1$ such that $C^{-1} B \leq A \leq C B$.

Let us note that yet another characterization of the Sobolev space has been obtained recently in [9] and [10].

The above theorem was a point of departure in [19] for the definition of a Sobolev space on an arbitrary metric space equipped with a locally finite Borel measure. For further results involving this approach see e.g. [3], [8], [13], [15], [16], [18], [20]-[22], [24]-[30], [32]-[37], [39], [44], [45], [49], [52], [53].

If $u \in W^{1, p}\left(\mathbb{R}^{n}\right), 1 \leq p<\infty$, then we have an elementary inequality ([7], [19])

$$
|u(x)-u(y)| \leq C(n)|x-y|\left(M_{2|x-y|}|\nabla u|(x)+M_{2|x-y|}|\nabla u|(y)\right) \quad \text { a.e. }
$$

where

$$
M_{R} g(x)=\sup _{r<R} f_{B(x, r)}|g(z)| d z
$$

is the restricted Hardy-Littlewood maximal function. Here and in what follows the integral average of a function $u$ over a set $E$ is denoted by

$$
u_{E}=f_{E} u d x=\frac{1}{|E|} \int_{E} u d x
$$

where $|E|$ denotes Lebesgue measure of $E$. Moreover $C$ will always stand for a general constant that can change its value even in the same string of estimates. Writing $C=C(n)$ we will emphasize that the constant depends on $n$ only.

If we take $R=\infty$ in the definition of $M_{R}$, then we obtain the classical Hardy-Littlewood maximal function

$$
M u(x)=\sup _{r>0} \underset{B(x, r)}{f}|u| .
$$

Hence it follows from inequality (2) that

$$
|u(x)-u(y)| \leq C|x-y|(M|\nabla u|(x)+M|\nabla u|(y)) \quad \text { a.e. }
$$

This and the boundedness of the maximal function in $L^{p}$ for $p>1$ (see [50]) imply (1) with $g=C M|\nabla u| \in L^{p}\left(\mathbb{R}^{n}\right)$. The implication in the opposite direction in Theorem 1 follows from the lemma.

Lemma 2 ([20, Proposition 1]; cf. [28, Remark 5.13]). If $u \in L_{\mathrm{loc}}^{1}\left(\mathbb{R}^{n}\right)$ and $0 \leq g \in L_{\mathrm{loc}}^{1}\left(\mathbb{R}^{n}\right)$ satisfy inequality (1) a.e., then $\nabla u \in L_{\mathrm{loc}}^{1}\left(\mathbb{R}^{n}\right)$ in 
the weak sense and

$$
|\nabla u| \leq C(n) g \quad \text { a.e. }
$$

This lemma is relatively easy and its proof is based on the observation that (1) implies absolute continuity of $u$ on almost all lines parallel to coordinate axes. If we know in addition that $g \in L^{p}\left(\mathbb{R}^{n}\right)$, then inequality (3) implies that $|\nabla u| \in L^{p}\left(\mathbb{R}^{n}\right)$, which completes the proof of Theorem 1 .

Observe that the only place in the proof of Theorem 1 where the assumption $p>1$ was employed was the application of the boundedness of the maximal function in $L^{p}$. It turns out that the assumption $p>1$ is essential because Theorem 1 does not hold for $p=1$. This follows from the next example.

ExAmple $3([20])$. Let $\Omega=(-1 / 2,1 / 2)$ and $u(x)=-x /(|x| \log |x|)$. Then $u \in W^{1,1}(\Omega)$ because $u^{\prime}(x)=|x|^{-1}(\log |x|)^{-2} \in L^{1}(\Omega)$. Suppose now that there exists $0 \leq g \in L^{1}(-1 / 2,1 / 2)$ such that (1) holds a.e. Then for a.e. $0<x<1 / 2$ we have $|u(x)-u(-x)| \leq 2 x(g(x)+g(-x))$ and hence

$$
\frac{-2}{\log x} \leq 2 x(g(x)+g(-x))
$$

which, in turn, yields

$$
\int_{-1 / 2}^{1 / 2} g(x) d x=\int_{0}^{1 / 2}(g(x)+g(-x)) d x \geq \int_{0}^{1 / 2} \frac{-d x}{x \log x}=\infty .
$$

This contradicts integrability of $g$. The function $u$ is defined on the interval $(-1 / 2,1 / 2)$ only, but one can extend it to a function in $W^{1,1}(\mathbb{R})$ to fit into the setting of Theorem 1.

The main result of the present paper is the following characterization of $W^{1,1}\left(\mathbb{R}^{n}\right)$.

THEOREM 4. $u \in W^{1,1}\left(\mathbb{R}^{n}\right)$ if and only if $u \in L^{1}\left(\mathbb{R}^{n}\right)$ and there exist $0 \leq g \in L^{1}\left(\mathbb{R}^{n}\right)$ and $\sigma \geq 1$ such that

$$
|u(x)-u(y)| \leq|x-y|\left(M_{\sigma|x-y|} g(x)+M_{\sigma|x-y|} g(y)\right) \quad \text { a.e. }
$$

Moreover if (4) holds, then $|\nabla u| \leq C(n, \sigma) g$ a.e.

The implication from left to right follows from the elementary inequality at (2). It turns out, however, that the implication from right to left is much more difficult than the corresponding one in Theorem 1.

The proof that inequality (4) implies $u \in W^{1,1}\left(\mathbb{R}^{n}\right)$ is split into two steps. In the first step we prove that (4) implies the family of Poincaré type inequalities

$$
f_{B}\left|u-u_{B}\right| \leq C r f_{3 \sigma B} g
$$


for every ball $B$ of any radius $r$. Here and in what follows, $3 \sigma B$ denotes the ball concentric with $B$ and with radius $3 \sigma$ times that of $B$. Observe that inequality (5) with $3 \sigma B$ replaced by $B$ would readily follow from (1) upon integration over $x, y \in B$. In our situation, however, we cannot integrate (4) because the maximal function of an $L^{1}$ function need not be integrable. This is the main difficulty in the proof and, actually, this first step is the main new ingredient in the proof.

In the second step we show that the family of inequalities (5) on every ball $B$ imply that $u \in W^{1,1}\left(\mathbb{R}^{n}\right)$ with $|\nabla u| \leq C g$ a.e. This implication has previously been proved in [15]. Here we provide a new, simpler proof.

Both steps are direct consequences of the following more general results applied to $p=1$. We will prove the lemmas in the whole generality, i.e. for all $1 \leq p<\infty$. This way we will clearly see what kind of new difficulties have to be faced when passing from the case $p>1$ to $p=1$.

Lemma 5. Let $u \in L_{\mathrm{loc}}^{1}\left(\mathbb{R}^{n}\right), 0 \leq g \in L_{\mathrm{loc}}^{p}\left(\mathbb{R}^{n}\right), 1 \leq p<\infty$, and $\sigma \geq 1$. Then the inequality

$$
|u(x)-u(y)| \leq|x-y|\left(\left(M_{\sigma|x-y|} g^{p}(x)\right)^{1 / p}+\left(M_{\sigma|x-y|} g^{p}(y)\right)^{1 / p}\right) \quad \text { a.e. }
$$

implies that

$$
f_{B}\left|u-u_{B}\right| \leq C(n, p, \sigma) r\left(f_{3 \sigma B} g^{p}\right)^{1 / p}
$$

for every ball $B$ of any radius $r$.

For $p>1$ Lemma 5 was proved in [24] and [29]. The case $p=1$ turns out to be much more difficult.

Lemma 6. Assume that $u \in L_{\mathrm{loc}}^{1}\left(\mathbb{R}^{n}\right), 0 \leq g \in L_{\mathrm{loc}}^{p}\left(\mathbb{R}^{n}\right), 1 \leq p<\infty$, and $\sigma \geq 1$ are such that

$$
f_{B}\left|u-u_{B}\right| \leq r\left(f_{\sigma B} g^{p}\right)^{1 / p}
$$

for every ball $B$ of any radius $r$. Then $u \in W_{\mathrm{loc}}^{1, p}\left(\mathbb{R}^{n}\right)$ and

$$
|\nabla u| \leq C(n) g \quad \text { a.e. }
$$

If $u \in W_{\text {loc }}^{1, p}\left(\mathbb{R}^{n}\right)$, then by the classical Poincaré inequality [14, p. 142], we have

$$
f_{B}\left|u-u_{B}\right| \leq C(n) r f_{B}|\nabla u| \leq C r\left(f_{\sigma B}|\nabla u|^{p}\right)^{1 / p},
$$

which shows that we also have the opposite implication in Lemma 6 , and thus (8) is a necessary and sufficient condition for $u$ to be in $W_{\text {loc }}^{1, p}\left(\mathbb{R}^{n}\right)$.

As already mentioned, the case $p=1$ of Lemma 6 was proved in [15]. The case $1<p<\infty$ was proved earlier in [36]. If we assume, however, that 
$1 \leq p<\infty$, and in addition $g \in L^{q}$ for some $q>p$, then Lemma 6 follows essentially from the work of Calderón [11].

Proof of Lemma 5. All the constants $C$ in the proof will depend on $n, p$ and $\sigma$ only. First let us sketch the proof for $p>1$. We will clearly see why this proof cannot be extended to the case $p=1$.

It follows from (6) that for $x, y \in B$,

$$
|u(x)-u(y)| \leq|x-y|\left(\left(M\left(g^{p} \chi_{3 \sigma B}\right)(x)\right)^{1 / p}+\left(M\left(g^{p} \chi_{3 \sigma B}\right)(y)\right)^{1 / p}\right) \quad \text { a.e. }
$$

Employing the Cavalieri principle we obtain

$$
\begin{aligned}
f_{B}\left|u-u_{B}\right| & \leq f_{B} f_{B}|u(x)-u(y)| d x d y \\
& \leq 4 r f_{B}\left(M\left(g^{p} \chi_{3 \sigma B}\right)(x)\right)^{1 / p} d x \\
& =4 r|B|^{-1} \int_{0}^{\infty}\left|\left\{x \in B ;\left(M\left(g^{p} \chi_{3 \sigma B}\right)(x)\right)^{1 / p}>t\right\}\right| d t \\
& =4 r|B|^{-1}\left(\int_{0}^{t_{0}}+\int_{t_{0}}^{\infty}\right) .
\end{aligned}
$$

For $0<t \leq t_{0}$ we estimate the integrand by the measure of the ball $B$, and for $t>t_{0}$ we estimate it by the weak type estimate for the maximal function (see [50]). This gives

$$
\begin{aligned}
f_{B}\left|u-u_{B}\right| & \leq 4 r|B|^{-1}\left(\int_{0}^{t_{0}}|B| d t+\int_{t_{0}}^{\infty}\left(\frac{C(n)}{t^{p}} \int_{3 \sigma B} g^{p}\right) d t\right) \\
& =4 r|B|^{-1}\left(t_{0}|B|+C(n, p) t_{0}^{1-p} \int_{3 \sigma B} g^{p}\right) .
\end{aligned}
$$

Now taking $t_{0}=\left(|B|^{-1} \int_{3 \sigma B} g^{p}\right)^{1 / p}$ yields the result. Observe that the assumption $p>1$ was employed to integrate $t^{-p}$ from $t_{0}$ to $\infty$.

Now assume that $p=1$. We will employ some ideas from the proof of the Sobolev embedding theorem in [19]. Fix a ball $B$. Replacing $u$ by $u-b$, where $b$ is any constant, will not affect inequalities (6) and (7). Hence by subtracting a suitable constant from $u$ we can assume that ess $\inf _{E}|u|=0$, where $E \subset B$ is any set of positive Lebesgue measure. The set $E$ will be chosen later.

For $x, y \in B$ we have

$$
|u(x)-u(y)| \leq|x-y|(M h(x)+M h(y)),
$$


where $h=g \chi_{3 \sigma B}$. Now it suffices to prove that

$$
f_{B}\left|u-u_{B}\right| \leq C r f_{3 \sigma B} h .
$$

If $h=0$ a.e. then $u$ is constant in $B$ and hence (10) follows. Thus we can assume that $h>0$ on a set of positive measure and hence $\int_{3 \sigma B} h>0$. Moreover we can assume that

$$
h \geq \frac{1}{2} f_{3 \sigma B} h>0 \text { on } 3 \sigma B \text { and } h=0 \text { on } \mathbb{R}^{n} \backslash 3 \sigma B,
$$

otherwise we replace $h$ by

$$
h+\left(f_{3 \sigma B} h\right) \chi_{3 \sigma B}
$$

We will prove that any integrable function $h$ with properties (9) and (11) satisfies (10) as well. For $k \in \mathbb{Z}$ let

$$
E_{k}=\left\{x \in B ; M h(x) \leq 2^{k}\right\} \quad \text { and } \quad a_{k}=\sup _{E_{k}}|u| .
$$

Since

$$
\int_{B}\left|u-u_{B}\right| \leq 2 \int_{B}|u| \leq 2 \sum_{k=-\infty}^{\infty} a_{k}\left|E_{k} \backslash E_{k-1}\right|
$$

we need to find good estimates for $a_{k}$ in order to estimate the left hand side of $(10)$.

From $E_{k} \subseteq E_{k+1}$ it follows that $a_{k} \leq a_{k+1}$. In order to estimate the growth of $a_{k}$ we have to estimate $a_{k}$ in terms of $a_{k-1}$ first. By (9) the function $u$ restricted to $E_{k}$ is $2^{k+1}$-Lipschitz. Hence for $x \in E_{k}$ and $y \in E_{k-1}$ we have

$$
|u(x)| \leq|u(x)-u(y)|+|u(y)| \leq 2^{k+1}|x-y|+a_{k-1} .
$$

To obtain a good estimate for the right hand side we have to show that for a given $x \in E_{k}$ there exists $y \in E_{k-1}$ with a relatively small distance to $x$, $|x-y|$. Choose $x \in E_{k}$ arbitrarily and observe that

$$
|B(x, r) \cap B| \geq \omega_{n}(r / 2)^{n} \quad \text { for } r \leq \operatorname{diam} B .
$$

Here $\omega_{n}$ denotes the volume of the unit ball. Assume that $\left|E_{k-1}\right|>0$. Choose $r<\operatorname{diam} B$ such that

$$
\omega_{n}(r / 2)^{n}>\left|B \backslash E_{k-1}\right| .
$$

Then (14) and (15) imply that there exists $y \in E_{k-1}$ such that $|x-y|<r$. Since the lower bound for $r$ satisfying (15) is $2 \omega_{n}^{-1 / n}\left|B \backslash E_{k-1}\right|^{1 / n}$ we conclude from (13) upon taking the supremum over $x \in E_{k}$ that

$$
a_{k} \leq a_{k-1}+C 2^{k}\left|B \backslash E_{k-1}\right|^{1 / n} .
$$


Invoking the weak type estimate for the maximal function (see [50]), we obtain

$$
\left|B \backslash E_{k-1}\right|=\left|\left\{x \in B ; M h(x)>2^{k-1}\right\}\right| \leq \frac{C}{2^{k}} \int_{3 \sigma B} h,
$$

and hence

$$
a_{k} \leq a_{k-1}+C 2^{k(1-1 / n)}\left(\int_{3 \sigma B} h\right)^{1 / n} .
$$

Assume now that $n \geq 2$. The case $n=1$ can be treated in a similar way, we leave the details to the reader.

Iterating this inequality yields

$$
\begin{aligned}
a_{k} & \leq a_{k_{0}}+C\left(\sum_{i=k_{0}+1}^{k} 2^{i(1-1 / n)}\right)\left(\int_{3 \sigma B} h\right)^{1 / n} \\
& \leq a_{k_{0}}+C 2^{k(1-1 / n)}\left(\int_{3 \sigma B} h\right)^{1 / n}
\end{aligned}
$$

for $k>k_{0}$, provided $\left|E_{k_{0}}\right|>0$. For $k \leq k_{0}$ we will use the estimate $a_{k} \leq a_{k_{0}}$. Choose $k_{0}$ such that

$$
\left|E_{k_{0}-1}\right|<|B| / 2 \leq\left|E_{k_{0}}\right|
$$

Such a $k_{0}$ exists because $E_{k}=\emptyset$ for sufficiently small $k$, due to the lower bound (11) for $h$, and $\left|E_{k}\right| \rightarrow|B|$ as $k \rightarrow \infty$. Since $E_{k_{0}} \neq \emptyset$, there exists $x \in B$ such that

$$
\frac{1}{2} f_{3 \sigma B} h \leq M h(x) \leq 2^{k_{0}} .
$$

The left inequality at (18) follows from (11). On the other hand the left inequality at (17) along with the weak type estimates for the maximal function implies

$$
\frac{|B|}{2}<\left|B \backslash E_{k_{0}-1}\right|=\left|\left\{x \in B ; M h(x)>2^{k_{0}-1}\right\}\right| \leq \frac{C}{2^{k_{0}}} \int_{3 \sigma B} h .
$$

The two inequalities (18) and (19) yield

$$
2^{k_{0}} \approx f_{3 \sigma B} h
$$

As mentioned at the beginning of the proof, we can assume that ess $\inf _{E_{k_{0}}}|u|$ $=0$. Since $u$ is $2^{k_{0}+1}$-Lipschitz on $E_{k_{0}}$ we have

$$
a_{k_{0}} \leq 2^{k_{0}+1} \operatorname{diam} B \leq C \operatorname{diam} B \underset{3 \sigma B}{f_{3 \sigma}} h .
$$


Now (12) together with the estimates (16), (20) and (21) implies

$$
\begin{aligned}
\int_{B} \mid u- & u_{B}\left|\leq 2 \sum_{k=-\infty}^{\infty} a_{k}\right| E_{k} \backslash E_{k-1} \mid \\
\leq & 2\left(\sum_{k=-\infty}^{k_{0}} a_{k_{0}}\left|E_{k} \backslash E_{k-1}\right|\right. \\
& \left.+\sum_{k=k_{0}+1}^{\infty}\left(a_{k_{0}}+C 2^{k(1-1 / n)}\left(\int_{3 \sigma B} h\right)^{1 / n}\right)\left|E_{k} \backslash E_{k-1}\right|\right) \\
\leq & 2\left(\sum_{k=-\infty}^{\infty} a_{k_{0}}\left|E_{k} \backslash E_{k-1}\right|+C\left(\int_{3 \sigma B} h\right)^{1 / n} \sum_{k=k_{0}+1}^{\infty} 2^{k(1-1 / n)}\left|B \backslash E_{k-1}\right|\right) \\
\leq & C \operatorname{diam} B\left(\int_{3 \sigma B} h\right)|B|+C\left(\int_{3 \sigma B} h\right)^{1 / n} \sum_{k=k_{0}+1}^{\infty} 2^{k(1-1 / n)} 2^{-k} \int_{3 \sigma B} h \\
\leq & C \operatorname{diam} B \int_{3 \sigma B} h,
\end{aligned}
$$

which completes the proof of Lemma 5 .

Proof of Lemma 6. At the beginning we will follow the argument of Calderón [11, Theorem 4] (cf. [19, proof of Theorem 1]); then, however, we have to use different ideas because Calderón's argument relies on the $L^{q / p}$ integrability of the maximal function of $g^{p}$ under the additional assumption that $g \in L^{q}$ for some $q>p$.

All the constants $C$ in the proof will depend on $n$ only.

Let $\psi \in C_{0}^{\infty}\left(B^{n}(0,1)\right)$ with $\psi \geq 0$ and $\int \psi=1$ be a generating mollifier. As usual we set $\psi_{\varepsilon}(x)=\varepsilon^{-n} \psi(x / \varepsilon)$ and consider a smooth approximation of $u$ defined by $u * \psi_{\varepsilon}$.

The distributional derivative $\partial u / \partial x_{i}, i=1, \ldots, n$, is a functional on $C_{0}^{\infty}\left(\mathbb{R}^{n}\right)$ defined by

$$
\frac{\partial u}{\partial x_{i}}[\varphi]:=-\int u \frac{\partial \varphi}{\partial x_{i}} \quad \text { for } \varphi \in C_{0}^{\infty}\left(\mathbb{R}^{n}\right) .
$$

Note that

$$
\frac{\partial u}{\partial x_{i}}[\varphi]=-\lim _{\varepsilon \rightarrow 0} \int\left(u * \psi_{\varepsilon}\right) \frac{\partial \varphi}{\partial x_{i}}=\lim _{\varepsilon \rightarrow 0} \int\left(u * \frac{\partial \psi_{\varepsilon}}{\partial x_{i}}\right) \varphi .
$$

Since $\int \partial \psi_{\varepsilon} / \partial x_{i}=0$ we conclude that

$$
\left(u * \frac{\partial \psi_{\varepsilon}}{\partial x_{i}}\right)(x)=\left(u-u_{B(x, \varepsilon)}\right) * \frac{\partial \psi_{\varepsilon}}{\partial x_{i}}(x)
$$


and hence

$$
\left|u * \frac{\partial \psi_{\varepsilon}}{\partial x_{i}}\right|(x) \leq C \int_{B(x, \varepsilon)}\left|u(y)-u_{B(x, r)}\right| d y \cdot \varepsilon^{-n-1} \leq C\left(\int_{B(x, \sigma \varepsilon)} g^{p}\right)^{1 / p} .
$$

For a compact set $K \subset \mathbb{R}^{n}$ we have

$$
\begin{aligned}
\int_{K}\left|u * \frac{\partial \psi_{\varepsilon}}{\partial x_{i}}\right|^{p} & \leq C^{p} \int_{K} f_{B(x, \sigma \varepsilon)} g^{p}(y) d y d x \\
& =C^{p} \omega_{n}^{-1}(\sigma \varepsilon)^{-n} \int_{K_{\sigma \varepsilon}} g^{p}(y) \int_{K} \chi_{B(y, \sigma \varepsilon)}(x) d x d y \\
& \leq C^{p} \int_{K_{\sigma \varepsilon}} g^{p}(y) d y
\end{aligned}
$$

where $K_{\sigma \varepsilon}$ is the set of points in $\mathbb{R}^{n}$ with distance to $K$ less than $\sigma \varepsilon$. Now (22), (23) and Hölder's inequality yield

$$
\begin{aligned}
\left|\frac{\partial u}{\partial x_{i}}[\varphi]\right| & \leq \liminf _{\varepsilon \rightarrow 0}\left(\int|\varphi|^{p^{\prime}}\right)^{1 / p^{\prime}}\left(\int_{\operatorname{supp} \varphi}\left|u * \frac{\partial \psi_{\varepsilon}}{\partial x_{i}}\right|^{p}\right)^{1 / p} \\
& \leq C\left(\int|\varphi|^{p^{\prime}}\right)^{1 / p^{\prime}}\left(\int_{\operatorname{supp} \varphi} g^{p}\right)^{1 / p}
\end{aligned}
$$

where $1 / p+1 / p^{\prime}=1$ with $p^{\prime}=\infty$ if $p=1$.

Assume for the time being that $1<p<\infty$. Fix a ball $B$. Then (24) applied to $\varphi \in C_{0}^{\infty}(B)$ implies that

$$
\varphi \mapsto \frac{\partial u}{\partial x_{i}}[\varphi]
$$

extends to a continuous functional in $\left(L^{p^{\prime}}(B)\right)^{*}=L^{p}(B)$. Hence $\partial u / \partial x_{i} \in$ $L^{p}(B)$ and

$$
\left(\int_{B}\left|\frac{\partial u}{\partial x_{i}}\right|^{p}\right)^{1 / p} \leq C\left(\int_{B} g^{p}\right)^{1 / p} .
$$

Inequality (26) applied to balls that converge to Lebesgue points readily shows that

$$
\left|\frac{\partial u}{\partial x_{i}}\right| \leq C g \quad \text { a.e. }
$$

In the case $p=1$ we have

$$
\left|\frac{\partial u}{\partial x_{i}}[\varphi]\right| \leq C\|\varphi\|_{\infty} \int_{\operatorname{supp} \varphi} g .
$$

Hence (25) extends to a continuous linear functional on $C_{0}\left(\mathbb{R}^{n}\right)$, the space of continuous functions vanishing at infinity. Thus according to the Riesz 
representation theorem [46],

$$
\frac{\partial u}{\partial x_{i}}[\varphi]=\int \varphi d \mu
$$

for some signed Radon measure $\mu$. We will show that $\mu$ is absolutely continuous with respect to the Lebesgue measure. By contradiction assume that there is a compact set $K$ of Lebesgue measure zero and such that $\mu(K) \neq 0$. Let $\varphi_{i} \in C_{0}^{\infty}\left(K_{1 / i}\right)$ with $0 \leq \varphi_{i} \leq 1$ and $\left.\varphi_{i}\right|_{K} \equiv 1$ be a decreasing sequence of functions. Here as before $K_{1 / i}$ stands for the $1 / i$-neighborhood of $K$. We have

$$
0<|\mu(K)| \leftarrow\left|\int \varphi_{i} d \mu\right| \leq C\left\|\varphi_{i}\right\|_{\infty} \int_{K_{1 / i}} g \rightarrow 0
$$

as $i \rightarrow \infty$, which is a contradiction. Thus according to the Radon-Nikodym theorem and the definition of the weak derivative we have

$$
d \mu=\frac{\partial u}{\partial x_{i}} d x, \quad \frac{\partial u}{\partial x_{i}} \in L_{\mathrm{loc}}^{1} .
$$

Hence inequality (27) implies that

$$
\left|\int \varphi \frac{\partial u}{\partial x_{i}}\right| \leq C\|\varphi\|_{\infty} \int_{B} g
$$

for every $\varphi \in C_{0}^{\infty}(B)$. Since the sign function $\operatorname{sgn}\left(\partial u / \partial x_{i}\right)$ can be approximated in $L^{1}$ by $\varphi \in C_{0}^{\infty}(B)$ with $\|\varphi\|_{\infty} \leq 1$ we conclude that

$$
\int_{B}\left|\frac{\partial u}{\partial x_{i}}\right| \leq C \int_{B} g
$$

for every ball $B$. Then the argument with Lebesgue points yields

$$
\left|\frac{\partial u}{\partial x_{i}}\right| \leq C g \quad \text { a.e. }
$$

This completes the proof of Lemma 6 and hence that of Theorem 4 .

If we put all the results together we obtain the following theorem as a direct consequence.

Theorem 7. For $u \in L^{p}\left(\mathbb{R}^{n}\right), 1 \leq p<\infty$, the following conditions are equivalent:

(i) $u \in W^{1, p}\left(\mathbb{R}^{n}\right)$.

(ii) There exists $0 \leq g \in L^{p}\left(\mathbb{R}^{n}\right)$ such that

$$
f_{B}\left|u-u_{B}\right| \leq r f_{B} g
$$

for every ball $B$ of any radius $r$. 
(iii) There exist $0 \leq g \in L^{p}\left(\mathbb{R}^{n}\right), 1 \leq q \leq p$ and $\sigma \geq 1$ such that

$$
f_{B}\left|u-u_{B}\right| \leq r\left(f_{\sigma B} g^{q}\right)^{1 / q}
$$

for every ball $B$ of any radius $r$.

(iv) There exist $0 \leq g \in L^{p}\left(\mathbb{R}^{n}\right), 1 \leq q \leq p$ and $\sigma \geq 1$ such that

$$
|u(x)-u(y)| \leq|x-y|\left(\left(M_{\sigma|x-y|} g^{q}(x)\right)^{1 / q}+\left(M_{\sigma|x-y|} g^{q}(y)\right)^{1 / q}\right) \quad \text { a.e. }
$$

Moreover each of the inequalities in (ii)-(iv) implies that

$$
|\nabla u| \leq C g \quad \text { a.e. }
$$

Observe that Theorem 1 is not included here. Theorem 7 extends to the case of a regular domain replacing $\mathbb{R}^{n}$. Part of the implications extend even to the more general setting of metric spaces equipped with a doubling measure (see [15], [22], [21], [24], [29], [36]). For a direct proof of the implication (iii) $\Rightarrow($ iv) see $[24$, Theorem 3.2].

\section{References}

[1] L. Ambrosio, Some fine properties of sets of finite perimeter in Ahlfors regular metric measure spaces, Adv. Math. 159 (2001), 51-67.

[2] L. Ambrosio and B. Kirchheim, Currents in metric spaces, Acta Math. 185 (2000), $1-80$.

[3] L. Ambrosio and P. Tilli, Selected topics on "Analysis on metric spaces", Scuola Norm. Sup., Pisa, 2000.

[4] E. Berkson, J. Bourgain, A. Pełczyński and M. Wojciechowski, Canonical Sobolev projections of weak type $(1,1)$, Mem. Amer. Math. Soc. 714 (2001).

[5] M. Biroli and U. Mosco, Sobolev inequalities on homogeneous spaces, Potential Anal. 4 (1995), 311-324.

[6] J. Björn, P. MacManus and N. Shanmugalingam, Fat sets and pointwise boundary estimates for p-harmonic functions in metric spaces, J. Anal. Math. 85 (2001), 339-369.

[7] B. Bojarski and P. Hajłasz, Pointwise inequalities for Sobolev functions and some applications, Studia Math. 106 (1993), 77-92.

[8] M. Bourdon et H. Pajot, Cohomologie $\ell_{p}$ et espaces de Besov, J. Reine Angew. Math. 558 (2003), 85-108.

[9] J. Bourgain, H. Brezis and P. Mironescu, Another look at Sobolev spaces, in: Optimal Control and Partial Differential Equations, (J. L. Menaldi, E. Rofman and A. Sulem (eds.), a volume in honor of A. Bensoussan's 60th birthday, IOS Press, 2001, 439455.

[10] H. Brezis, How to recognize constant functions. A connection with Sobolev spaces, Uspekhi Mat. Nauk 57 (2002), no. 4, 59-74 (in Russian).

[11] A. P. Calderón, Estimates for singular integral operators in terms of maximal functions, Studia Math. 44 (1972), 563-582.

[12] J. Cheeger, Differentiability of Lipschitz functions on metric measure spaces, Geom. Funct. Anal. 9 (1999), 428-517. 
[13] D. Danielli, N. Garofalo and D. M. Nhieu, Non-doubling Ahlfors measures, perimeter measures, and the characterization of the trace spaces of Sobolev functions in Carnot-Carathéodory spaces, Mem. Amer. Math. Soc., to appear.

[14] L. C. Evans and R. F. Gariepy, Measure Theory and Fine Properties of Functions, Stud. Adv. Math., CRC Press, Boca Raton, FL, 1992.

[15] B. Franchi, P. Hajłasz and P. Koskela, Definitions of Sobolev classes on metric spaces, Ann. Inst. Fourier (Grenoble) 49 (1999), 1903-1924.

[16] B. Franchi, G. Z. Lu and R. L. Wheeden, Representation formulas and weighted Poincaré inequalities for Hörmander vector fields, Internat. Mat. Res. Notices 1996, no. $1,1-14$.

[17] N. Garofalo and D.-M. Nhieu, Isoperimetric and Sobolev inequalities for CarnotCarathéodory spaces and the existence of minimal surfaces, Comm. Pure Appl. Math. 49 (1996), 1081-1144.

[18] V. Gol'dshtein and M. Troyanov, Axiomatic theory of Sobolev spaces, Exposition. Math. 19 (2001), 289-336.

[19] P. Hajłasz, Sobolev spaces on an arbitrary metric space, Potential Anal. 5 (1996), 403-415.

[20] - Geometric approach to Sobolev spaces and badly degenerated elliptic equations, in: Nonlinear Analysis and Applications (Warszawa, 1994), N. Kenmochi et al. (eds.), GAKUTO Internat. Ser. Math. Sci. Appl. 7, Gakkōtosho, Tokyo, 1996, 141-168.

[21] P. Hajłasz, Sobolev spaces on metric-measure spaces, Contemp. Math., to appear.

[22] P. Hajłasz and J. Kinnunen, Hölder quasicontinuity of Sobolev functions on metric spaces, Rev. Mat. Iberoamericana 14 (1998), 601-622.

[23] P. Hajłasz and P. Koskela, Sobolev meets Poincaré, C. R. Acad. Sci. Paris Sér. I Math. 320 (1995), 1211-1215.

[24] —, —, Sobolev met Poincaré, Mem. Amer. Math. Soc. 688 (2000).

[25] P. Hajłasz and O. Martio, Traces of Sobolev functions on fractal type sets and characterization of extension domains, J. Funct. Anal. 143 (1997), 221-246.

[26] Y. S. Han and D. C. Yang, New characterizations and applications of inhomogeneous Besov and Triebel-Lizorkin spaces on homogeneous type spaces and fractals, Dissertationes Math. 403 (2002).

[27] P. Harjulehto, Maximal inequality in $(s, m)$-uniform domains, Ann. Acad. Sci. Fenn. Math. 27 (2002), 291-306.

[28] J. Heinonen, Lectures on Analysis on Metric Spaces, Universitext, Springer, New York, 2001.

[29] J. Heinonen and P. Koskela, Quasiconformal maps on metric spaces with controlled geometry, Acta Math. 181 (1998), 1-61.

[30] J. Heinonen, P. Koskela, N. Shanmugalingam and J. T. Tyson, Sobolev classes of Banach space-valued functions and quasiconformal mappings, J. Anal. Math. 85 (2001), 87-139.

[31] J. Heinonen and D. Sullivan, On the locally branched Euclidean metric gauge, Duke Math. J. 114 (2002), 15-41.

[32] A. Kałamajska, On compactness of embedding for Sobolev spaces defined on metric spaces, Ann. Acad. Sci. Fenn. 24 (1999), 123-132.

[33] T. Kilpeläinen, J. Kinnunen and O. Martio, Sobolev spaces with zero boundary values on metric spaces, Potential Anal. 12 (2000), 233-247.

[34] J. Kinnunen and V. Latvala, Lebesgue points for Sobolev functions on metric spaces, Rev. Mat. Iberoamericana 18 (2002), 685-700.

[35] J. Kinnunen and O. Martio, The Sobolev capacity on metric spaces, Ann. Acad. Sci. Fenn. Math. 21 (1996), 367-382. 
[36] P. Koskela and P. MacManus, Quasiconformal mappings and Sobolev spaces, Studia Math. 131 (1998), 1-17.

[37] M. Kronz, Some function spaces on spaces of homogeneous type, Manuscripta Math. 106 (2001), 219-248.

[38] Y. P. Liu, G. Z. Lu and R. L. Wheeden, Some equivalent definitions of high order Sobolev spaces on stratified groups and generalizations to metric spaces, Math. Ann. 323 (2002), 157-174.

[39] P. Ola, L. Päivärinta and V. Serov, Recovering singularities from backscattering in two dimensions, Comm. Partial Differential Equations 26 (2001), 697-715.

[40] A. Pełczyński and K. Senator, On isomorphisms of anisotropic Sobolev spaces with "classical Banach spaces" and a Sobolev type embedding theorem, Studia Math. 84 (1986), 169-218.

[41] A. Pełczyński and M. Wojciechowski, Contribution to the isomorphic classification of Sobolev spaces $L_{(k)}^{p}(\Omega)(1 \leq p<\infty)$, in: Recent Progress in Functional Analysis (Valencia, 2000), North-Holland Math. Stud. 189, North-Holland, Amsterdam, 2001, 133-142.

[42] - - -, Molecular decompositions and embedding theorems for vector-valued Sobolev spaces with gradient norm, Studia Math. 107 (1993), 61-100.

[43] - - - Sobolev spaces in several variables in $L^{1}$-type norms are not isomorphic to Banach lattices, Ark. Mat. 40 (2002), 363-382.

[44] J. Rissanen, Wavelets on self-similar sets and the structure of the spaces $M(E, \mu)$, Ann. Acad. Sci. Fenn. Math. Diss. 125 (2002).

[45] A. S. Romanov, On a generalization of Sobolev spaces, Sibirsk. Mat. Zh. 39 (1998), 949-953 (in Russian); English transl.: Siberian Math. J. 39 (1998), 821-824.

[46] W. Rudin, Real and Complex Analysis, 3rd ed., McGraw-Hill, New York, 1987.

[47] S. Semmes, Finding curves on general spaces through quantitative topology, with applications to Sobolev and Poincaré inequalities, Selecta Math. (N.S.) 2 (1996), 155-295.

[48] —, Some Novel Types of Fractal Geometry, Oxford Math. Monographs, The Clarendon Press, Oxford Univ. Press, New York, 2001.

[49] N. Shanmugalingam, Newtonian spaces: an extension of Sobolev spaces to metric measure spaces, Rev. Mat. Iberoamericana 16 (2000), 243-279.

[50] E. Stein, Singular Integrals and Differentiability Properties of Functions, Princeton Math. Ser. 30, Princeton Univ. Press, Princeton, NJ, 1970

[51] K. T. Sturm, Analysis on local Dirichlet spaces III. The parabolic Harnack inequality, J. Math. Pures Appl. 75 (1996), 273-297.

[52] M. Troyanov, Approximately Lipschitz mappings and Sobolev mappings between metric spaces, in: Proc. Analysis and Geometry (Novosibirsk, 1999), Izdat. Ross. Akad. Nauk Sib. Otd. Inst. Mat., Novosibirsk, 2000, 585-594 (in Russian).

[53] M. Troyanov and V. Gol'dshtein, An integral characterization of Hajłasz-Sobolev space, C. R. Acad. Sci. Paris Sér. I Math. 333 (2001), 445-450.

Institute of Mathematics

Warsaw University

Banacha 2

02-097 Warszawa, Poland

E-mail: hajlasz@mimuw.edu.pl 